\title{
ALGORITMO DE ORGANIZAÇÃO DAS EQUAÇÕES MATRICIAIS OBTIDAS DA ANÁLISE POR INSPEÇÃO EM UM CIRCUITO ELÉTRICO COM FONTES DE TENSÃO E DE CORRENTE, DEPENDENTES OU INDEPENDENTES ${ }^{1}$
}

\author{
Carlos Renato Borges dos Santos ${ }^{2}$
}

\begin{abstract}
RESUMO
Este artigo apresenta um algoritmo para organização e solução das equações obtidas pelos métodos de análise por inspeção nodal ou por malhas. Oferece, também, uma rápida revisão sobre os métodos de inspeção, que funcionam como uma ferramenta de rápido equacionamento do circuito elétrico, que podem ser utilizados genericamente para circuitos elétricos lineares, com fontes de tensão e de corrente, dependentes e independentes, simplificando o equacionamento, sem riscos de desenvolver equações linearmente dependentes. Um circuito elétrico pode ser solucionado de diversas formas, e os métodos de equacionamento dependem de algumas características específicas do circuito. Este trabalho mostra que os métodos de inspeção podem ser utilizados genericamente em circuitos elétricos lineares com fontes de tensão e de corrente, dependentes, ou independentes. Em contrapartida, a presença de diferentes tipos de fontes pode gerar um sistema de equações que precisam ser manipuladas para que se tornem um sistema linear do tipo $A \cdot x=b$, o que foi realizado a partir do desmembramento matricial dos elementos do sistema. Utilizou-se o Matlab para determinar o sistema linear no qual as incógnitas pudessem ser determinadas, aplicando-se o algoritmo proposto neste trabalho.
\end{abstract}

Palavras-chave: Circuitos elétricos. Algoritmo. Sistema linear. Inspeção.

\section{INTRODUÇÃO}

A aplicação do método dos nós envolvendo fontes de tensão (dependentes ou independentes) implica, normalmente, na aplicação de supernós. Um supernó é formado envolvendo-se uma fonte de tensão conectada entre dois nós. São propriedades de um supernó: a fonte de tensão dentro do supernó fornece uma equação de restrição necessária para encontrar as tensões nodais; um supernó não tem nenhuma tensão própria; requer a aplicação tanto da LKC (Lei de Kirchhoff das correntes) como da LKT (Lei de Kirchhoff das tensões). Por outro lado, se aplicado o método das malhas num circuito que contenha fontes de corrente (dependentes ou

\section{Como citar este artigo:}

SANTOS, C. R. B. dos. Algoritmo de organização das equações matriciais obtidas da análise por inspeção em circuito elétrico com fontes de tensão e de corrente, dependentes ou independentes. ForScience: revista científica do IFMG, Formiga, v. 6, n. 1, e00336, jan.jun. 2018.

2 Autor para correspondência: Carlos Renato Borges dos Santos, IFMG - Campus Formiga, carlos.renato@ifmg.edu.br 
SANTOS, C. R. dos. Algoritmo de organização das equações matriciais obtidas da análise por inspeção em um circuito elétrico com fontes de tensão e de corrente, dependentes ou independentes

independentes), utiliza-se, normalmente, a supermalha. Uma supermalha é aplicada em duas malhas que possuam uma fonte de corrente em comum. São propriedades da supermalha: a fonte de corrente na supermalha fornece a equação de restrição necessária para encontrar as correntes de malha; uma supermalha não possui corrente própria; requer a aplicação da LKT, bem como da LKC (ALEXANDER; SADIKU, 2013).

\subsection{Sistema linear}

A análise de circuitos elétricos necessita da composição de um conjunto de equações lineares utilizando-se as leis de Kirchhoff e de Ohm. Metodologias sistematizadas são essenciais para selecionar um conjunto adequado de variáveis independentes (COSTA, 2013, p.43).

Equações lineares podem ser solucionadas por sistemas matriciais do tipo mostrado na Equação (1). O número de equações implica na ordem do sistema linear e corresponde ao número de incógnitas do sistema. Para fontes independentes, o número de equações corresponde ao número de malhas para LKT, ou ao número do nós (exceto o de referência) para LKC (JOHNSON; HILBURN; JOHNSON, 1993; IRWIN, 2008; COSTA, 2013).

$$
A_{n, n} \cdot x_{n, 1}=b_{n, 1}
$$

As matrizes do sistema linear da Equação (1) estão organizadas na forma que cada método descreve. No caso da LKT, a matriz $A$ é a de resistências, $x$ representa as correntes de malha e $b$ representa as fontes de tensão (os elementos desconhecidos do sistema); no caso da LKC, a matriz $A$ é a matriz de condutâncias, $x$ representa as tensões nodais (os elementos desconhecidos do sistema) e $b$, as fontes de corrente. Em todo caso, essas matrizes são facilmente montadas quando há somente fontes de tensão ou de corrente no circuito elétrico. A matriz $A$ pode perder a simetria quando há fontes dependentes (IRWIN, 2008).

Deve-se suplementar as equações das tensões de nó com equações de restrição impostas pela presença das fontes dependentes. Da mesma forma, deve-se suplementar as equações de correntes de malha, num circuito que contenha fontes dependentes, pelas equações de restrições adequadas (NILSSON; RIEDEL, 2009, p.69 e 75).

\subsection{Métodos de inspeção}

Os métodos de inspeção são apresentados em diversos livros didáticos de circuitos elétricos, tais como Irwin (2008), Edminister (1991), Costa (2013) e Yaro e Lyra (2006). As tensões e correntes de um circuito são obtidas a partir da solução de sistemas lineares do tipo mostrado na Equação (1). O metódo uttilizado, na prática, será aquele que resultar no menor número 
de equações, observada a restrição da aplicação do método das malhas em circuitos planares (COSTA, 2013, p.59; ALEXANDER; SADIKU, 2013, p.92). O método dos nós, em muitos casos, é mais fácil de ser aplicado (JOHNSON; HILBURN; JOHNSON, 1993, Cap.6).

A inspeção de circuitos é realizada aplicando-se o método das malhas ou o método dos nós. No método das malhas, a diagonal principal da matriz $A$ é obtida pela soma das resistências de cada malha; já os demais elementos dessa matriz são obtidos pelos resistores em comum nas malhas, com sinal negativo. O vetor $x$ representa as correntes de malha. Os elementos do vetor $b$ representam as tensões das fontes, sejam elas de tensão (valor conhecido) ou de corrente (desconhecido).

No método dos nós, a diagonal principal da matriz $A$ é obtida pela soma das condutâncias ligadas ao nó correspondente, enquanto que no restante da matriz os elementos são obtidos pelas condutâncias conectadas aos nós dos índices da matriz. O vetor $x$ representa as tensões nodais. Os elementos do vetor $\mathrm{b}$ representam as correntes das fontes, sejam elas de corrente (valor conhecido) ou de tensão (corrente desconhecida).

Uma vez consideradas as afirmações, e obedecendo às características intrínsecas de cada tipo de fonte, para o equacionamento do sistema utilizando-se a inspeção, uma fonte de corrente pode ser considerada como uma fonte cuja tensão seja desconhecida (incógnita) e com corrente conhecida; logo, uma fonte de tensão pode ser considerada uma fonte cuja corrente seja desconhecida (incógnita), mas de tensão conhecida. Portanto, não é necessária a distinção que restrinja a utilização da inspeção nodal ou por malha.

\section{EXEMPLOS DE CIRCUITO}

São apresentados três circuitos para exemplificar os algoritmos de organização dos elementos das matrizes do sistema linear. Em cada circuito, são mostradas as análises por inspeção nodal e por malhas.

\subsection{Circuito 1}

O circuito 1 é mostrado na Figura 1.

A Figura 2 apresenta as malhas 1, 2 e 3 para aplicação da LKT e os nós 1, 2 e 3 para aplicação da LKC. 
SANTOS, C. R. dos. Algoritmo de organização das equações matriciais obtidas da análise por inspeção em um circuito elétrico com fontes de tensão e de corrente, dependentes ou independentes

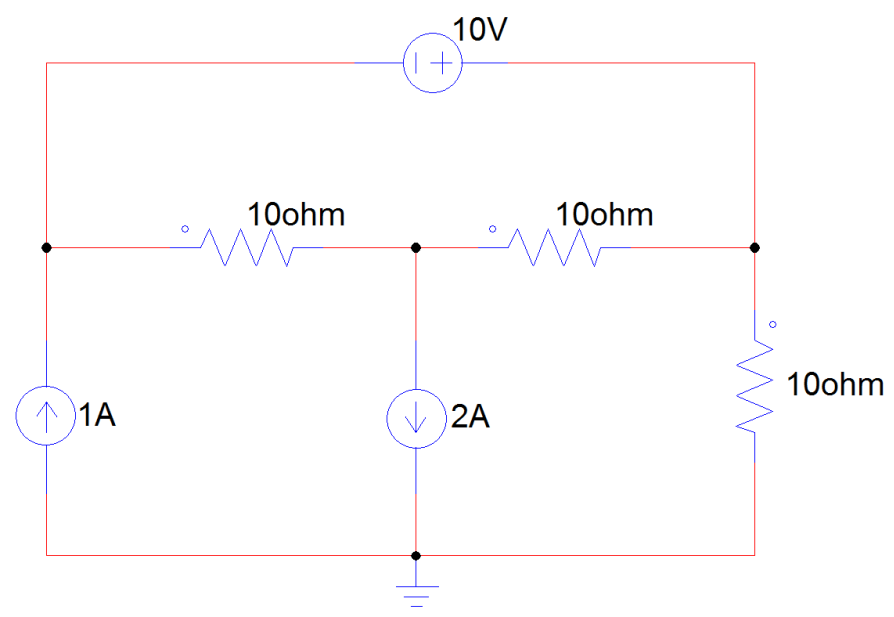

Figura 1 - Análise por malhas e por nós do circuito 2 Fonte: do autor

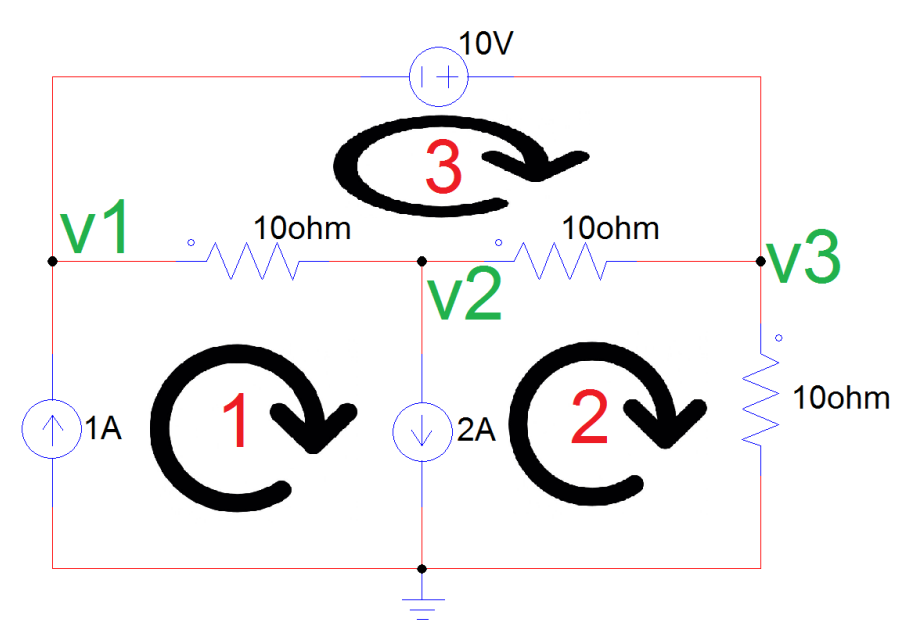

Figura 2 - Análise por malhas e por nós do circuito 2 Fonte: do autor

Inspeção nodal

Utilizando o método dos nós, a tensão nodal do nó 3 é dada por $v_{3}=v_{1}+10$. A corrente da fonte de tensão é desconhecida, dada por $i_{v_{10 V}}$. Por inspeção nodal, tem-se a Equação (2).

$$
\left[\begin{array}{ccc}
\frac{1}{10} & -\frac{1}{10} & 0 \\
-\frac{1}{10} & \frac{1}{5} & -\frac{1}{10} \\
0 & -\frac{1}{10} & \frac{1}{5}
\end{array}\right] \cdot\left[\begin{array}{c}
v_{1} \\
v_{2} \\
v_{1}+10
\end{array}\right]=\left[\begin{array}{c}
1-i_{v_{10 V}} \\
-2 \\
i_{v_{10 V}}
\end{array}\right]
$$

ForSci.: r. cient. IFMG, Formiga, v. 6, n. 1, e00336, jan./jun. 2018. 
SANTOS, C. R. dos. Algoritmo de organização das equações matriciais obtidas da análise por inspeção em um circuito elétrico com fontes de tensão e de corrente, dependentes ou independentes

Inspeção de malhas

Utilizando o método das malhas, a corrente de malha da malha $1\left(I_{1}=1 A\right)$ e a corrente de malha da malha $2\left(I_{1}-I_{2}=2\right)$ é, então, dada por $I_{2}=I_{1}-2=1-2=-1 A$. As tensões das fontes de corrente são desconhecidas, $v_{i_{1 A}}$ e $v_{i_{2 A}}$. A Equação (3) mostra o sistema por inspeção de malhas.

$$
\left[\begin{array}{ccc}
10 & 0 & -10 \\
0 & 20 & -10 \\
-10 & -10 & 20
\end{array}\right] \cdot\left[\begin{array}{c}
1 \\
-1 \\
I_{3}
\end{array}\right]=\left[\begin{array}{c}
v_{i_{1 A}}+v_{i_{2 A}} \\
-v_{i_{2 A}} \\
10
\end{array}\right]
$$

\subsection{Circuito 2}

O circuito da Figura 3 apresenta uma fonte de tensão dependente de tensão. Este circuito apresenta 3 nós e 3 malhas.

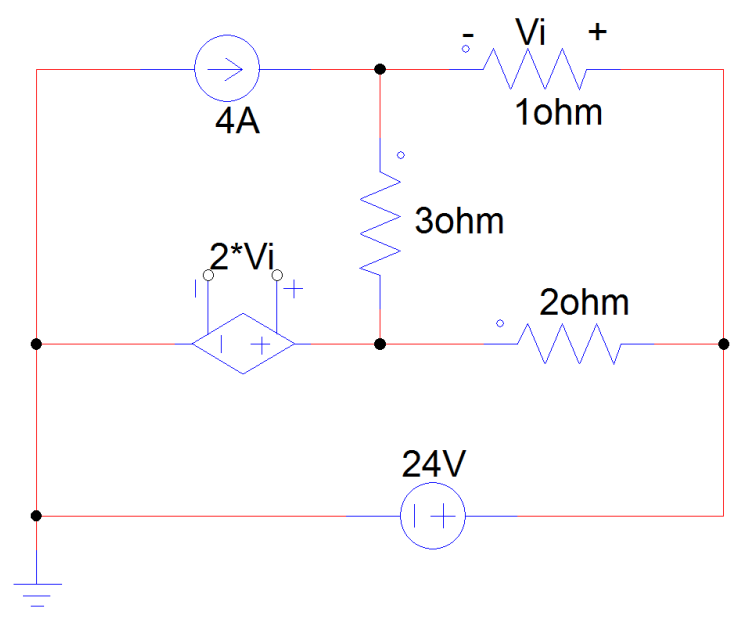

Figura 3 - Circuito 2

Fonte: (IRWIN, 2008), com adaptações

A Figura 4 apresenta as malhas 1, 2 e 3, e os nós 1, 2 e 3.

Inspeção nodal

$\mathrm{Na}$ inspeção nodal, as fontes de tensão são consideradas como fontes de correntes (de valores desconhecidos). Logo, a corrente da fonte de tensão de $24 \mathrm{~V}$ é expressa por $i_{v_{24 V}}$ e a fonte de tensão dependente é expressa por $i_{v_{d e p}}$. A tensão do nó 1 é conhecida (24 V), a equação 


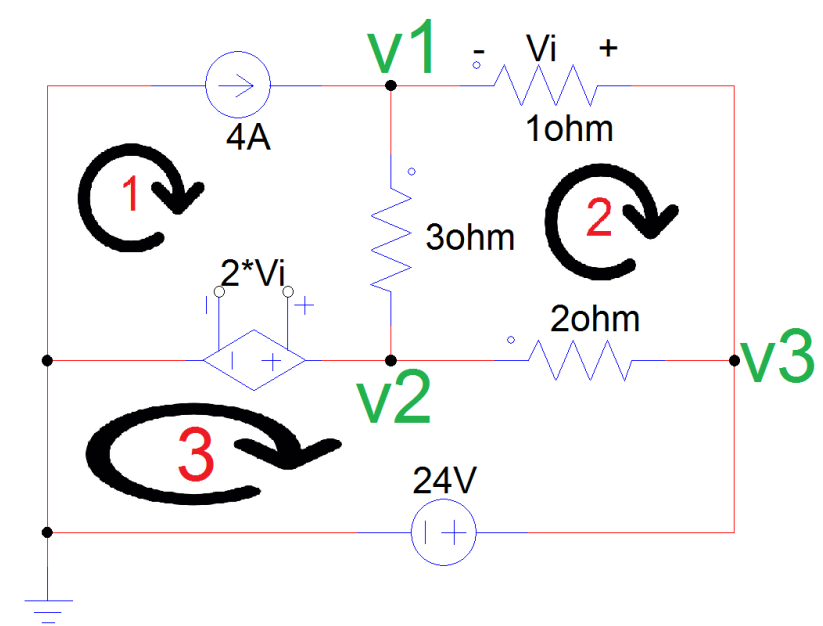

Figura 4 - Circuito 3

Fonte: (IRWIN, 2008), com adaptações

de restrição necessária, devido à fonte dependente, é dada por $V i=v_{3}-v_{1}=24-v_{1}$. Dessa forma, tem-se a Equação (4).

$$
\left[\begin{array}{ccc}
\frac{4}{3} & -\frac{1}{3} & -1 \\
-\frac{1}{3} & \frac{5}{6} & -\frac{1}{2} \\
-1 & -\frac{1}{2} & \frac{3}{2}
\end{array}\right] \cdot\left[\begin{array}{c}
v_{1} \\
2 \cdot\left(24-v_{1}\right) \\
24
\end{array}\right]=\left[\begin{array}{c}
4 \\
i_{v_{d e p}} \\
i_{v_{24 V}}
\end{array}\right]
$$

Inspeção de malhas

Analisando a Figura 4, para a aplicação da LKT, deve-se considerar a fonte de corrente de $4 A$ como uma fonte de tensão desconhecida $v_{i_{4 A}}$. Como equação de restrição da fonte dependente, $I_{2}=-\frac{V i}{1}$, logo: $V i=-I_{2}$. A equação do circuito é mostrada em (5).

$$
\left[\begin{array}{ccc}
3 & -3 & 0 \\
-3 & 6 & -2 \\
0 & -2 & 2
\end{array}\right] \cdot\left[\begin{array}{c}
4 \\
I_{2} \\
I_{3}
\end{array}\right]=\left[\begin{array}{c}
v_{i_{4 A}}-2 \cdot\left(-I_{2}\right) \\
0 \\
-24+2 \cdot\left(-I_{2}\right)
\end{array}\right]
$$

\subsection{Circuito 3}

Haffner (2005) apresenta um circuito com uma pequena diferença dos circuitos apresentados neste trabalho. No circuito da Figura 5, foi dada a tensão de $8 \mathrm{~V}$ (corrente de ramo de 4 A) no resistor de $2 \Omega$, desconhecendo-se o valor da fonte de tensão $V_{x}$. A diferença, nesse caso, é que não se conhece a tensão, nem mesmo a corrente da fonte $V_{x}$. 
SANTOS, C. R. dos. Algoritmo de organização das equações matriciais obtidas da análise por inspeção em um circuito elétrico com fontes de tensão e de corrente, dependentes ou independentes

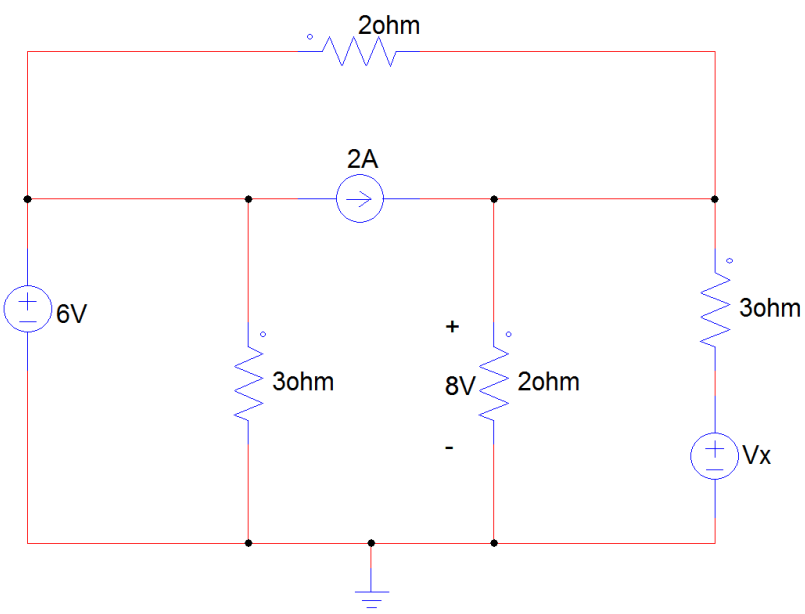

Figura 5 - Circuito 3

Fonte: (HAFFNER, 2005), com adaptações

A Figura 6 apresenta o circuito com os nós 1, 2 e 3. Destaca-se que o nó 3 poderia ser eliminado se a fonte de tensão $V_{x}$ e o resistor de $3 \Omega$ fossem convertidos em uma fonte de corrente. Neste caso, para demonstrar a metodologia proposta neste trabalho, optou-se por manter o nó 3. Por inspeção nodal, obtém-se a Equação (6).

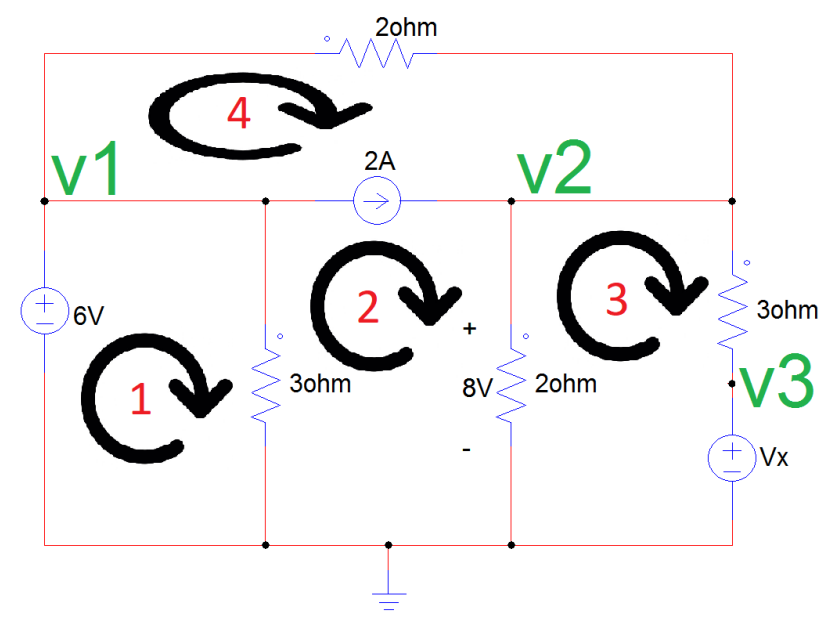

Figura 6 - Circuito 3

Fonte: (HAFFNER, 2005), com adaptações

ForSci.: r. cient. IFMG, Formiga, v. 6, n. 1, e00336, jan./jun. 2018. 
Inspeção nodal

A Equação (6) é obtida pela inspeção nodal.

$$
\left[\begin{array}{ccc}
\frac{5}{6} & -\frac{1}{2} & 0 \\
-\frac{1}{2} & \frac{4}{3} & -\frac{1}{3} \\
0 & -\frac{1}{3} & \frac{1}{3}
\end{array}\right] \cdot\left[\begin{array}{c}
6 \\
8 \\
v_{x}
\end{array}\right]=\left[\begin{array}{c}
i_{v_{6 V}}-2 \\
2 \\
i_{x}
\end{array}\right]
$$

Inspeção por malhas

O circuito possui 4 malhas, logo o sistema possui dimensão 4. Após a inspeção por malha, tem-se a Equação (7).

$$
\left[\begin{array}{cccc}
3 & -3 & 0 & 0 \\
-3 & 5 & -2 & 0 \\
0 & -2 & 5 & 0 \\
0 & 0 & 0 & 2
\end{array}\right] \cdot\left[\begin{array}{c}
I_{1} \\
I_{2} \\
I_{2}-4 \\
I_{2}-2
\end{array}\right]=\left[\begin{array}{c}
6 \\
v_{i_{2 A}} \\
-v_{x} \\
-v_{i_{2 A}}
\end{array}\right]
$$

\section{MATERIAL E MÉTODOS}

O sistema linear descrito na Equação (1), obtido por inspeção, não apresenta todas as variáveis do sistema contidas no vetor $x$. Da mesma forma, todos os termos independentes não estão contidos no vetor $b$ quando há fontes de tensão e de corrente no circuito.

Observando a Equação (1), o sistema de matrizes que produz o vetor $x$ é mostrado na Equação (8), e o sistema de matrizes que produz o vetor $b$ é mostrado na Equação (9).

$$
x_{n, 1}=\left[X_{\text {coef }}\right]_{n, n} \cdot\left[x_{v a r}\right]_{n, 1}+\left[c_{x}\right]_{n, 1}
$$

em que:

$\left[x_{v a r}\right]_{n, 1} \Rightarrow$ Variáveis do sistema $\Rightarrow\left[\begin{array}{llll}x_{1} & x_{2} & \ldots & x_{n}\end{array}\right]^{T}$;

$\left[X_{\text {coef }}\right]_{n, n} \Rightarrow$ Matriz de coeficientes das variáveis de $x$ da Equação (1);

$\left[c_{x}\right]_{n, 1} \Rightarrow$ Constantes em $x$ (são fontes independentes de tensão na LKC ou fontes de corrente na LKT).

$$
b_{n, 1}=\left[B_{\text {coef }}\right]_{n, n} \cdot\left[x_{\text {var }}\right]_{n, 1}+\left[c_{b}\right]_{n, 1}
$$

em que:

$\left[B_{\text {coef }}\right]_{n, n} \Rightarrow$ matriz de coeficientes das variáveis em $b$ (da Equação (1));

$\left[c_{b}\right]_{n, 1} \Rightarrow$ constantes em $b$ (fontes independentes de tensão na LKT ou fontes indepentes de corrente na LKC). 
SANTOS, C. R. dos. Algoritmo de organização das equações matriciais obtidas da análise por inspeção em um circuito elétrico com fontes de tensão e de corrente, dependentes ou independentes

Reescrevendo a Equação (1) a partir das Equações (8) e (9), tem-se a Equação (10).

$$
[A]_{n, n} \cdot\left(\left[X_{\text {coef }}\right]_{n, n} \cdot\left[x_{\text {var }}\right]_{n, 1}+\left[c_{x}\right]_{n, 1}\right)=\left[B_{\text {coef }}\right]_{n, n} \cdot\left[x_{\text {var }}\right]_{n, 1}+\left[c_{b}\right]_{n, 1}
$$

A Equação 11 elimina os parêntesis da Equação (10).

$$
[A]_{n, n} \cdot\left[X_{\text {coef }}\right]_{n, n} \cdot\left[x_{v a r}\right]_{n, 1}+[A]_{n, n} \cdot\left[c_{x}\right]_{n, 1}=\left[B_{\text {coef }}\right]_{n, n} \cdot\left[x_{v a r}\right]_{n, 1}+\left[c_{b}\right]_{n, 1}
$$

A Equação (12) isola o vetor de variáveis $x_{\text {var }}$.

$$
\left([A]_{n, n} \cdot\left[X_{\text {coef }}\right]_{n, n}-\left[B_{\text {coef }}\right]_{n, n}\right) \cdot\left[x_{\text {var }}\right]_{n, 1}=\left[c_{b}\right]_{n, 1}-[A]_{n, n} \cdot\left[c_{x}\right]_{n, 1}
$$

$\mathrm{Na}$ Equação (13) são mostradas as expressões do algoritmo de determinação dos vetores $b^{\prime}, x^{\prime}$ e da matriz $A^{\prime}$.

$$
\left\{\begin{array}{c}
A_{n, n}^{\prime}=[A]_{n, n} \cdot\left[X_{\text {coef }}\right]_{n, n}-\left[B_{\text {coef }}\right]_{n, n} \\
x_{n, 1}^{\prime}=\left[x_{v a r}\right]_{n, 1} \\
b_{n, 1}^{\prime}=\left[c_{b}\right]_{n, 1}-[A]_{n, n} \cdot\left[c_{x}\right]_{n, 1}
\end{array}\right.
$$

Após a execução dos algoritmos, o sistema linear contém matriz e vetores com a ordenação de seus elementos, prontos para a resolução do sistema $A^{\prime} \cdot x^{\prime}=b^{\prime}$.

Utilizou-se o Matlab para solucionar as expressões da Equação (13), através do algoritmo 1.

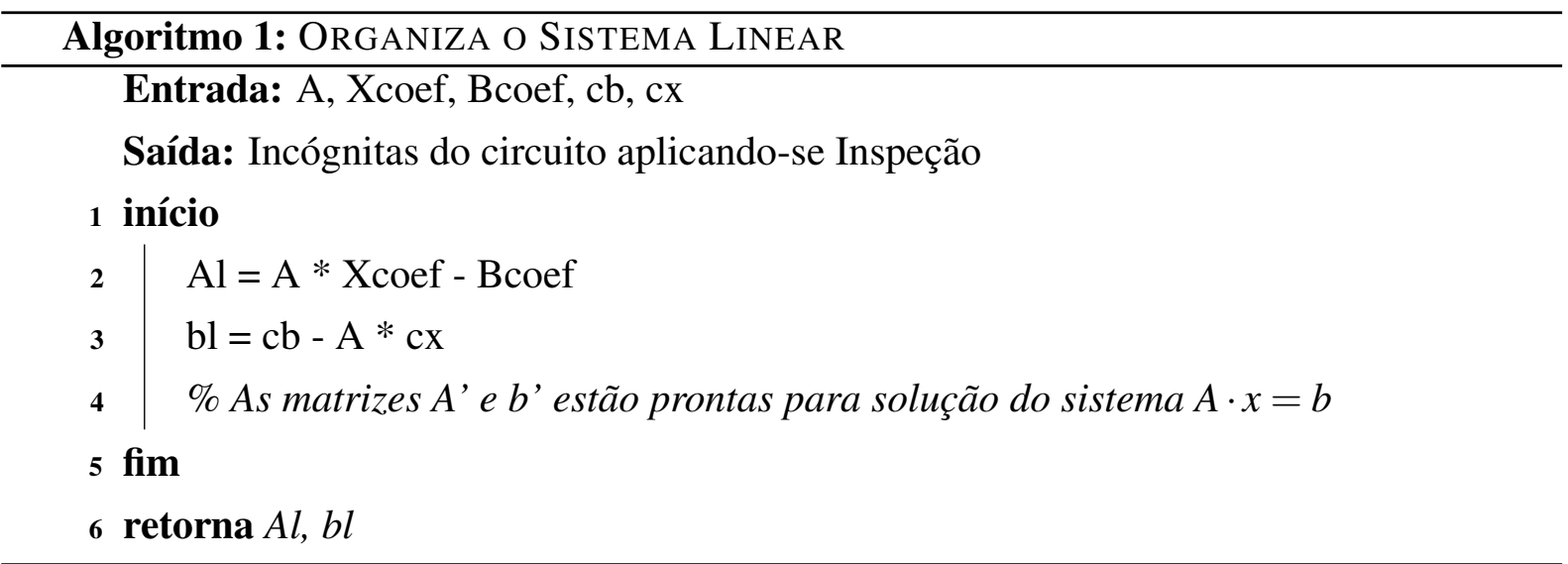

\section{RESULTADOS}

Exemplos de circuitos foram mostrados na seção 2. A organização dos elementos para a obtenção do sistema linear de cada circuito é mostrada a seguir. Nesta seção são mostrados os resultados do algoritmo de organização dos sistemas apresentados. 


\subsection{Circuito 1}

O circuito 1 é mostrado na Figura 2.

Método dos nós

O sistema decomposto é mostrado na Equação (14).

$$
\left[\begin{array}{ccc}
\frac{1}{10} & -\frac{1}{10} & 0 \\
-\frac{1}{10} & \frac{1}{5} & -\frac{1}{10} \\
0 & -\frac{1}{10} & \frac{1}{5}
\end{array}\right] \cdot\left(\left[\begin{array}{ccc}
1 & 0 & 0 \\
0 & 1 & 0 \\
1 & 0 & 0
\end{array}\right] \cdot\left[\begin{array}{c}
v_{1} \\
v_{2} \\
i_{v_{10 V}}
\end{array}\right]+\left[\begin{array}{c}
0 \\
0 \\
10
\end{array}\right]\right)=\left[\begin{array}{ccc}
0 & 0 & -1 \\
0 & 0 & 0 \\
0 & 0 & 1
\end{array}\right] \cdot\left[\begin{array}{c}
v_{1} \\
v_{2} \\
i_{v_{10 V}}
\end{array}\right]+\left[\begin{array}{c}
1 \\
-2 \\
0
\end{array}\right]
$$

Após a execução do algoritmo 1, tem-se o sistema linear da Equação (15).

$$
\left[\begin{array}{ccc}
0,1 & -0,1 & 1 \\
-0,2 & 0,2 & 0 \\
0.2 & -0,1 & -1
\end{array}\right] \cdot\left[\begin{array}{c}
v_{1} \\
v_{2} \\
i_{v_{10 V}}
\end{array}\right]=\left[\begin{array}{c}
1 \\
-1 \\
-2
\end{array}\right]
$$

\section{Método das malhas}

O sistema decomposto é mostrado na Equação (16).

$$
\left[\begin{array}{ccc}
10 & 0 & -10 \\
0 & 20 & -10 \\
-10 & -10 & 20
\end{array}\right] \cdot\left(\left[\begin{array}{lll}
0 & 0 & 0 \\
0 & 0 & 0 \\
0 & 0 & 1
\end{array}\right] \cdot\left[\begin{array}{c}
v_{i_{1 A}} \\
v_{i_{2 A}} \\
I_{3}
\end{array}\right]+\left[\begin{array}{c}
1 \\
-1 \\
0
\end{array}\right]\right)=\left[\begin{array}{ccc}
1 & 1 & 0 \\
0 & -1 & 0 \\
0 & 0 & 0
\end{array}\right] \cdot\left[\begin{array}{c}
v_{i_{1 A}} \\
v_{i_{2 A}} \\
I_{3}
\end{array}\right]+\left[\begin{array}{c}
0 \\
0 \\
10
\end{array}\right]
$$

Após a execução do algoritmo 1, tem-se o sistema linear da Equação (17).

$$
\left[\begin{array}{ccc}
-1 & -1 & -10 \\
0 & 1 & -10 \\
0 & 0 & 20
\end{array}\right] \cdot\left[\begin{array}{c}
v_{i_{1 A}} \\
v_{i_{2 A}} \\
I_{3}
\end{array}\right]=\left[\begin{array}{c}
-10 \\
20 \\
10
\end{array}\right]
$$

\subsection{Circuito 2}

O circuito 2 é mostrado na Figura 4. 
SANTOS, C. R. dos. Algoritmo de organização das equações matriciais obtidas da análise por inspeção em um circuito elétrico com fontes de tensão e de corrente, dependentes ou independentes

Método dos nós

A decomposição dos vetores e matrizes é mostrada na Equação (19).

$$
\begin{aligned}
& {\left[\begin{array}{ccc}
\frac{4}{3} & -\frac{1}{3} & -1 \\
-\frac{1}{3} & \frac{5}{6} & -\frac{1}{2} \\
-1 & -\frac{1}{2} & \frac{3}{2}
\end{array}\right] \cdot\left(\left[\begin{array}{ccc}
1 & 0 & 0 \\
-2 & 0 & 0 \\
0 & 0 & 0
\end{array}\right] \cdot\left[\begin{array}{c}
v_{1} \\
i_{v_{d e p}} \\
i_{v_{24 V}}
\end{array}\right]+\left[\begin{array}{c}
0 \\
48 \\
24
\end{array}\right]\right)=\left[\begin{array}{lll}
0 & 0 & 0 \\
0 & 1 & 0 \\
0 & 0 & 1
\end{array}\right] \cdot\left[\begin{array}{c}
v_{1} \\
i_{v_{\text {dep }}} \\
i_{v_{24 V}}
\end{array}\right]+\left[\begin{array}{l}
4 \\
0 \\
0
\end{array}\right]} \\
& {\left[\begin{array}{ccc}
\frac{4}{3} & -\frac{1}{3} & -1 \\
-\frac{1}{3} & \frac{5}{6} & -\frac{1}{2} \\
-1 & -\frac{1}{2} & \frac{3}{2}
\end{array}\right] \cdot\left(\left[\begin{array}{ccc}
1 & 0 & 0 \\
-2 & 0 & 0 \\
0 & 0 & 0
\end{array}\right] \cdot\left[\begin{array}{c}
v_{1} \\
i_{v_{d e p}} \\
i_{v_{24 V}}
\end{array}\right]+\left[\begin{array}{c}
0 \\
48 \\
24
\end{array}\right]\right)=\left[\begin{array}{lll}
0 & 0 & 0 \\
0 & 1 & 0 \\
0 & 0 & 1
\end{array}\right] \cdot\left[\begin{array}{c}
v_{1} \\
i_{v_{d e p}} \\
i_{v_{24 V}}
\end{array}\right]+\left[\begin{array}{l}
4 \\
0 \\
0
\end{array}\right]}
\end{aligned}
$$

\section{Método das malhas}

A decomposição de matrizes e de vetores da Equação (5) é mostrada na Equação (20).

$$
\left[\begin{array}{ccc}
3 & -3 & 0 \\
-3 & 6 & -2 \\
0 & -2 & 2
\end{array}\right] \cdot\left(\left[\begin{array}{lll}
0 & 0 & 0 \\
0 & 1 & 0 \\
0 & 0 & 1
\end{array}\right] \cdot\left[\begin{array}{c}
v_{x} \\
I_{2} \\
I_{3}
\end{array}\right]+\left[\begin{array}{l}
4 \\
0 \\
0
\end{array}\right]\right)=\left[\begin{array}{ccc}
1 & 2 & 0 \\
0 & 0 & 0 \\
0 & -2 & 0
\end{array}\right] \cdot\left[\begin{array}{c}
v_{x} \\
I_{2} \\
I_{3}
\end{array}\right]+\left[\begin{array}{c}
0 \\
0 \\
-24
\end{array}\right]
$$

O sistema linear resultante do algoritmo 1 é mostrado na Equação (21).

$$
\left[\begin{array}{ccc}
-1 & -5 & 0 \\
0 & 6 & -2 \\
0 & 0 & 2
\end{array}\right] \cdot\left[\begin{array}{c}
v_{x} \\
I_{2} \\
I_{3}
\end{array}\right]=\left[\begin{array}{c}
-12 \\
12 \\
-24
\end{array}\right]
$$

\subsection{Circuito 3}

O circuito 3 é mostrado na Figura 6.

Método dos nós

Para o circuito 3 foi utilizado apenas o método dos nós, já que a utilização da LKT formaria um sistema com dimensões maiores do que o LKC. A Equação (22) mostra o sistema decomposto.

$$
\left[\begin{array}{ccc}
\frac{5}{6} & -\frac{1}{2} & 0 \\
-\frac{1}{2} & \frac{4}{3} & -\frac{1}{3} \\
0 & -\frac{1}{3} & \frac{1}{3}
\end{array}\right] \cdot\left(\left[\begin{array}{lll}
0 & 0 & 0 \\
0 & 0 & 0 \\
0 & 1 & 0
\end{array}\right] \cdot\left[\begin{array}{c}
i_{x} \\
v_{x} \\
i_{v_{6 V}}
\end{array}\right]+\left[\begin{array}{l}
6 \\
8 \\
0
\end{array}\right]\right)=\left[\begin{array}{lll}
1 & 0 & 0 \\
0 & 0 & 0 \\
0 & 0 & 1
\end{array}\right] \cdot\left[\begin{array}{c}
i_{x} \\
v_{x} \\
i_{v_{6 V}}
\end{array}\right]+\left[\begin{array}{c}
-2 \\
2 \\
0
\end{array}\right]
$$


O sistema linear resultante, após a plicação do algoritmo 1, é mostrado na Equação (23).

$$
\left[\begin{array}{ccc}
-1 & 0 & 0 \\
0 & -\frac{1}{3} & 0 \\
0 & \frac{1}{3} & -1
\end{array}\right] \cdot\left[\begin{array}{c}
i_{x} \\
v_{x} \\
i_{v_{6 V}}
\end{array}\right]=\left[\begin{array}{c}
-3 \\
-5,6667 \\
2,6667
\end{array}\right]
$$

Método das malhas

A decomposição da Equação (7) é dada pelas Equações (24), (25) e (26).

$$
\begin{gathered}
A=\left[\begin{array}{cccc}
3 & -30 & 0 & 0 \\
-3 & 5 & -2 & 0 \\
0 & -2 & 5 & 0 \\
0 & 0 & 0 & 2
\end{array}\right] \\
x=X_{\text {coef }} \cdot x_{\text {var }}+c_{x}=\left[\begin{array}{cccc}
1 & 0 & 0 & 0 \\
0 & 1 & 0 & 0 \\
0 & 1 & 0 & 0 \\
0 & 1 & 0 & 0
\end{array}\right] \cdot\left[\begin{array}{c}
I_{1} \\
I_{2} \\
v_{i_{2 A}} \\
v_{x}
\end{array}\right]+\left[\begin{array}{c}
0 \\
0 \\
-4 \\
-2
\end{array}\right] \\
b=B_{\text {coef }} \cdot x_{\text {var }}+c_{b}=\left[\begin{array}{cccc}
0 & 0 & 0 & 0 \\
0 & 0 & 1 & 0 \\
0 & 0 & 0 & -1 \\
0 & 0 & -1 & 0
\end{array}\right] \cdot\left[\begin{array}{c}
I_{1} \\
I_{2} \\
v_{i_{2 A}} \\
v_{x}
\end{array}\right]+\left[\begin{array}{l}
6 \\
0 \\
0 \\
0
\end{array}\right]
\end{gathered}
$$

O sistema resultante após a execução do algoritmo 1 é mostrado na Equação (27).

$$
\left[\begin{array}{cccc}
3 & -3 & 0 & 0 \\
-3 & 3 & -1 & 0 \\
0 & 3 & 0 & 1 \\
0 & 2 & 1 & 0
\end{array}\right] \cdot\left[\begin{array}{c}
I_{1} \\
I_{2} \\
v_{i_{2 A}} \\
v_{x}
\end{array}\right]+\left[\begin{array}{c}
6 \\
-8 \\
20 \\
4
\end{array}\right]
$$

\section{CONCLUSÕES}

Este trabalho mostrou que os métodos de inspeção podem ser utilizados genericamente em circuitos elétricos lineares, obtendo-se um sistema de equações que precisam ser trabalhados para possibilitar seu solucionamento. Utilizou-se o Matlab para testar o algoritmo, a partir dos exemplos descritos. Dessa forma, equacionar o circuito tornou-se mais simples, sem particularidades que dificultam a determinação de um conjunto de equações linearmente independentes. 
SANTOS, C. R. dos. Algoritmo de organização das equações matriciais obtidas da análise por inspeção em um circuito elétrico com fontes de tensão e de corrente, dependentes ou independentes

A metodologia apresentada possui a vantagem de não exigir do usuário um conhecimento aprofundado em diversas técnicas de circuitos elétricos, tais como as teorias da substituição, sobreposição, supernós, supermalhas, além de outras. Por outro lado, a metodologia apresentada exige um esforço computacional maior, devido à decomposição de matrizes.

\title{
MATRIX EQUATIONS ORGANIZATION ALGORITHM OBTAINED FROM INSPECTION ANALYSIS IN AN ELECTRICAL CIRCUIT WITH VOLTAGE AND CURRENT SOURCES, DEPENDENT OR INDEPENDENT
}

\begin{abstract}
This paper presents an algorithm for the organization and solution of the equations obtained by nodal or mesh inspection methods. It also offers a quick review of inspection methods, which act as a tool for equationing the electrical circuit, which can be used generically for linear electric circuits, with independent and dependent voltage and current sources, simplifying the equation without risks of developing linearly dependent equations. An electrical circuit can be solved in several ways, and methods of equationing dependent on some specific characteristics of the circuit. This work shows which methods of inspection can be used generically in linear electric circuits with voltage and current sources, dependent or independent. In contrast, a presence of different types of sources can generate a system of equations that need to be manipulated to become a linear system of type $A \cdot x=b$, which was performed from the dismemberment elements of the system. Matlab was used to determine the linear system, in which the unknowns could be determined, applying the algorithm proposed in the work.
\end{abstract}

Keywords: Electric circuits. Algorithm. Linear system. Inspection method.

\section{REFERÊNCIAS}

ALEXANDER, C. K.; SADIKU, M. N. O. Fundamentos de Circuitos Elétricos. 5. ed. [S.1.: s.n.], 2013. 894 p.

COSTA, V. M. da. Circuitos Elétricos Lineares: enfoques teórico e prático. [S.1.]: Interciência LTDA, 2013. 542 p.

EDMINISTER, A. J. Circuitos Elétricos. 2. ed. São Paulo: Makron Books, 1991. 585 p.

HAFFNER, S. Análise de Circuitos I. Aula 4 Circuitos Elétricos Resistivos. Universidade Federal do Rio Grande do Sul, 2005. Disponível em: <http://www.ufrgs.br/eng04030/Aulas/ pdf\%20Haffner/aula04.pdf>. Acesso em: 15 ago 2017.

IRWIN, J. D. Análise de Circuitos em Engenharia. 4. ed. [S.1.]: Pearson, 2008. 866 p. 
SANTOS, C. R. dos. Algoritmo de organização das equações matriciais obtidas da análise por inspeção em um circuito elétrico com fontes de tensão e de corrente, dependentes ou independentes

JOHNSON, D. E.; HILBURN, J. L.; JOHNSON, J. R. Fundamentos de análise de circuitos elétricos. 4. ed. [S.1.]: Prentice Hall do Brasil, 1993. 552 p.

NILSSON, J. W.; RIEDEL, S. A. Circuitos Elétricos. 8. ed. [S.1.]: Pearson Education do Brasil, 2009.

YARO, B. J.; LYRA, A. C. C. Circuitos Elétricos. [S.1.]: Pearson Prentice Hall, 2006.

\section{DADOS DO AUTOR}

\section{Carlos Renato Borges dos Santos}

Lattes: http://lattes.cnpq.br/4669261214745681

E-mail: carlos.renato@ifmg.edu.br

Possui graduação em engenharia elétrica pela Universidade Federal de Goiás (2003), mestrado em engenharia elétrica e de Computação pela Universidade Federal de Goiás (2005) e doutorado em engenharia agrícola pela Universidade Federal de Lavras (2017), com doutoradosanduíche pela Universidade de Versalhes Saint Quentin en Yvelines (UVSQ). Atualmente é professor do Instituto Federal Minas Gerais. Atuou entre 2010 e 2012 no projeto de cooperação internacional (Brasil-França) em educação profissional e tecnológica na área da indústria eletroeletrônica 